\title{
Optimization of heterogeneous systems with Al planning heuristics and machine learning: a performance and energy aware approach
}

\author{
Suejb Memeti ${ }^{1}$ (D) Sabri Pllana ${ }^{2}$
}

Received: 12 February 2021 / Accepted: 20 September 2021 / Published online: 19 October 2021

(c) The Author(s) 2021

\begin{abstract}
Heterogeneous computing systems provide high performance and energy efficiency. However, to optimally utilize such systems, solutions that distribute the work across host CPUs and accelerating devices are needed. In this paper, we present a performance and energy aware approach that combines AI planning heuristics for parameter space exploration with a machine learning model for performance and energy evaluation to determine a near-optimal system configuration. For data-parallel applications our approach determines a near-optimal host-device distribution of work, number of processing units required and the corresponding scheduling strategy. We evaluate our approach for various heterogeneous systems accelerated with GPU or the Intel Xeon Phi. The experimental results demonstrate that our approach finds a near-optimal system configuration by evaluating only about $7 \%$ of reasonable configurations. Furthermore, the performance per Joule estimation of system configurations using our machine learning model is more than $1000 \times$ faster compared to the system evaluation by program execution.
\end{abstract}

Keywords Heterogeneous computing - Optimization - Artificial intelligence (AI) Machine learning (ML) · Planning heuristics

Mathematics Subject Classification 90C59 - 68T20 - 68W10

$\bowtie \quad$ Suejb Memeti

suejb.memeti@bth.se

Sabri Pllana

sabri.pllana@1nu.se

1 Department of Computer Science, Blekinge Tekniska Högskola, 37179 Karlskrona, Sweden

2 Department of Computer Science and Media Technology, Linnaeus University, 35195 Växjö, Sweden 


\section{Introduction}

Accelerators (such as, GPU or Intel Xeon Phi) are often used collaboratively with general-purpose CPUs to increase the overall system performance and improve energy efficiency. Currently, three of the top five most powerful computers in the TOP500 list [1] are heterogeneous computers that use GPUs as accelerators. Efficient programming of heterogeneous systems and splitting the work between the CPUs and accelerators are domains of particular interest [2-4].

Due to different architectural characteristics and the large number of system parameter configurations (such as, the number of threads, thread affinity, workload partitioning between multi-core processors of the host and the accelerating devices), achieving a good workload distribution that results in optimal performance and energy efficiency on heterogeneous systems is a non-trivial task [5,6]. An optimal system configuration that results with the highest throughput may not necessarily be the most energy efficient. Furthermore, the optimal system configuration is most likely to change for different types of applications, input problem sizes, and available resources.

Figure 1 depicts the process of system performance tuning. Traditionally the process of finding the optimal system parameters involved many iterations of selecting parameter values, program execution, and performance analysis (Fig. 1a). A bruteforce search requires the program execution for all parameter values of interest, and consequently for real-world programs and systems it may take an unreasonable long time to find the optimum. In contrast to brute-force search, an AI heuristic search [7,8] enables to find a near-optimum solution with fewer performance experiments based on nature-inspired algorithms (for instance, simulated annealing [9] or Artificial Bee Colony [10]). Figure $1 \mathrm{~b}$ depicts our approach that combines AI heuristic parameter value selection with a machine learning model for performance evaluation. The advantage of our approach is twofold:

- heuristic parameter value selection reduces significantly the number of performance experiments compared to brute-force search;

- system performance evaluation using a model is usually much faster than the program execution on a real-world system; moreover, availability of the system under study during the performance optimization process is not required once that the model has been developed.

In [11] authors provide a comprehensive review of literature that describes various approaches for using heuristics or machine learning for optimization of parallel computing systems from single-node systems [12,13] to large scale Grid and Cloud computing infrastructures [14-16]. Related work approaches for system optimization focus on utilizing either heuristics [17] or machine learning [12]. There is insufficient research in combining these two methods in particular for optimization of heterogeneous computing systems. Preliminary results of our optimization approach are discussed in [18]. However, [18] was limited to only performance optimization of systems accelerated with the Intel Xeon Phi. This paper extends our previous work as follows, 


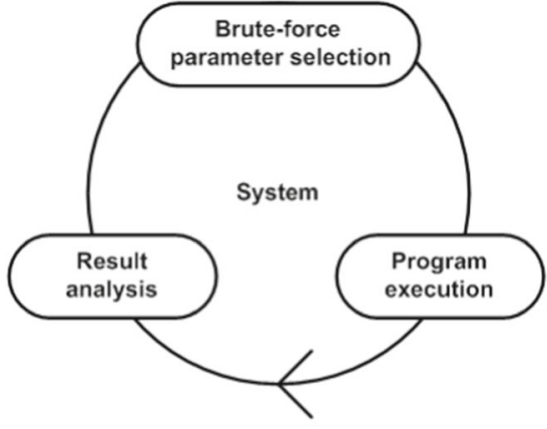

(a) Brute-force search

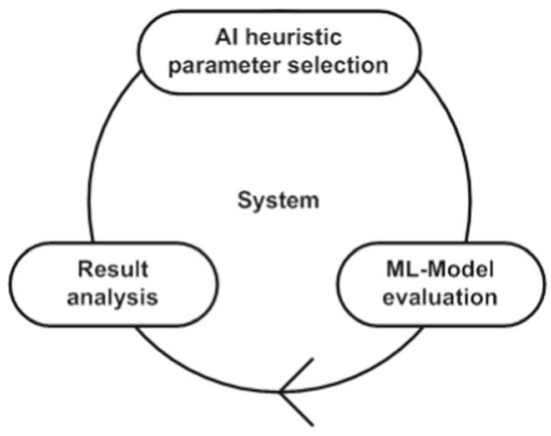

(b) AI heuristic search

Fig. 1 System performance tuning. a Evaluation of all possible configurations with program execution, b AI heuristic selection of candidate configurations and model-based evaluation

- we present a new machine learning model that estimates the performance per Joule of the system (that is, it considers also energy consumption, beside the performance);

- we demonstrate that our approach is general-applicable and it is not limited to Intel Xeon Phi, by applying our method also to GPU accelerated systems;

- we demonstrate the applicability of our approach with two applications: Pearson correlation coefficient and parallel pattern matching.

We use an AI heuristic search technique for parameter space exploration. The optimization process involves generation of system configuration based on random selection of parameter values and the system performance evaluation using a machine learning model. Instead of using a brute-force method to iterate through all possible parameter configurations, our method guides the process of intelligent navigation through the parameter space towards the determination of the near-optimal system configuration. As a result, our method requires only a small fraction of possible performance experiments. The empirical evaluation of our approach demonstrates that by using only about $7 \%$ of the total brute-force experiments we are able to determine a near-optimal system configuration with respect to the performance per Joule. Moreover, using our machine learning model for evaluation of system configurations is more than $1000 \times$ faster than the system evaluation by program execution.

This paper is organized as follows. We discuss the related research in Sect. 2. Section 3 introduces computing systems and applications that we use to illustrate our approach. We describe our optimization approach in Sect. 4 and experimentally evaluate it in Sect. 5. Section 6 provides a summary of the paper and a description of the future work.

\section{Related work}

In this section, first we discuss related research that addresses optimization of heterogeneous computing systems. We are interested to know whether the optimization 
involves host CPUs and device accelerators (such as, GPU or Intel Xeon Phi), AI heuristics are used for searching for optimal system parameters, machine learning is used for optimization, and whether the optimization goals are power/ energy consumption and performance. A summary of related work is provided in Table 1. Thereafter, we briefly describe how our approach that is presented in this paper differs from the related work.

Khaleghzadeh et al. [19] propose two algorithms to address the multi-objective optimization problem of heterogeneous parallel computing systems by considering the energy and the execution time. One of the algorithms considers the dynamic energy only and the other one considers the total energy of applications. The authors evaluate their algorithm for finding a global Pareto optimal solution on two different heterogeneous platforms, one accelerated with a GPU system and the other with Intel Xeon Phi. The proposed approach is evaluated using two different data-parallel application, including matrix multiplication and $2 \mathrm{D}$ fast Fourier transform. The authors show that the static energy (i.e. the energy consumption while the processing units are idle) in high performance computing centers is not negligible, and that the proposed solution is able to determine the Pareto optimal system configuration.

Manumachu and Lastovetsky [20] proposed a similar approach for optimization of homogeneous multi-core systems by considering the performance and energy consumption.

Cabrera et al. [21] propose a heuristic-based technique to improve the energy efficiency (i.e. energy consumption and the elapsed time) of heterogeneous systems with multiple different GPU accelerators by balancing the workload across various parallel processes in an iterative manner. While the authors have shown that the proposed technique yields to considerable improvements on heterogeneous systems accelerated with multiple-GPUs, the application of their method to homogeneous systems or heterogeneous systems that consider the host resources is left for future.

In a previous paper, Cabrera et al. [22] tackle the challenge of optimal workload distribution across multiple GPUs of a heterogeneous systems by employing machine learning. The authors focus on optimizing the energy consumption, however for the tested system there was a strong correlation between the execution time and energy consumption, which means that the fastest execution time was the most optimal with respect to energy consumption as well.

Huang et al. [23] propose a strategy for improvement of GPU power consumption based on dynamic voltage and frequency scaling (DVFS). They developed a DVFS model for energy optimization based on proportional-integral-derivative neural network (PIDNN). Three NVIDIA GPUs are used for experimental evaluation: Quadro FX 380, GTX 460, GTX 680. Authors have applied their method only to single GPU systems, and they plan to study multi-GPU systems in future.

Haidar et al. [24] study the relationship between energy consumption and performance for various BLAS kernels on Intel Xeon Phi Knights Landing (KNL). Authors use the PAPI library for power and performance monitoring. A conclusion of this study is that to achieve a good performance with minimal power consumption it is important to maximize the use of MCDRAM memory on KNL device and minimize the use of DDR4 RAM of the system. 
In [13], Kasichayanula et al. propose a software approach for real-time energy consumption measurements and analysis on individual GPU components. They use small subset of real-time statistics, and are able to infer about the activity of other micro-components using a linear-regression based prediction model.

Pereira et al. [17] propose an approach for determining optimal CUDA block dimension for execution of a wind field calculation program on GPU. Authors use particle swarm optimization (PSO) heuristic to find the CUDA block dimension that results with minimal program execution time. Using PSO they achieve $2 \times$ speed up compared to the previous GPU version of the program.

Hong and Kim [25] proposed an integrated energy and performance prediction model for GPU accelerators that is able to predict the optimal number of processors for a given memory-bound application that results with the peak performance and lowest energy consumption. Their experimental results with five various memory bound application benchmarks on a GPU architecture show up to $10 \%$ reduction of energy consumption.

Cerotti et al. [26] study the performance modeling of GPU accelerated computing systems. Authors present a language for description of system components and workload elements. Properties of system components are described with a set of parameters. For modeling systems that comprise CPUs and GPUs authors use queuing networks.

Benkner et al. [27] developed PEPPHER that is a programming framework for heterogeneous systems that comprise CPUs and accelerators (such as, GPU or Intel Xeon Phi). PEPPHER involves source-to-source compilation and a run-time system capable of mapping code components on an extensible set of target processor architectures. To address the performance portability, PEPPHER uses optimized component implementation variants for each execution context. Regression analysis of historical performance data from previous component executions is used to continuously learn associated performance models for run-time scheduling decisions.

The distribution of computation in heterogeneous systems accelerated with GPU devices for energy optimization has been studied by Ge et al. [28]. Authors propose the so called PEACH model that distributes the computations by splitting the workload between host and accelerators, and adaptively schedules these computations with regards to the computation units speed and energy consumption. Authors use analytical models to optimally determine the workload distribution and scheduling that aims at minimal energy consumption and best performance.

Ravi and Agrawal [29] propose a dynamic scheduling framework for heterogeneous computing systems that comprise various processing elements (such as, CPU or GPU). Authors specifically address data parallel loops. Scheduling decisions consider architectural trade-offs, computation and communication patterns. Performance optimization aims at minimizing data transfer between CPU and GPU, reducing the number of kernel invocation on GPU, and reducing the idle time of resources.

Grewe and O'Boyle [12] study task partitioning for OpenCL programs. Authors address task partitioning and mapping on heterogeneous systems that comprise a CPU and a GPU. Static analysis is used for code features extraction (such as, int and float operations, or data transfer size), and thereafter a machine learning model that is developed based on support vector machines (SVM) is used to map code features to 
work partition. The model predicts the percentages of work that should be assigned to GPU and CPU to achieve optimal performance.

Compared to the related work summarized in Table 1, in addition to using machine learning for modeling and evaluation of performance and power consumption, we use heuristics to search for a near-optimal system configuration. In a large parameter space for system configuration, heuristics have advantages (such as, time to solution or general applicability) compared to time-prohibitive brute-force approaches that aim at considering all possible configurations or compared to ad hoc approaches that are limited to a specific case.

\section{Background and motivation}

In this section, we describe heterogeneous computing systems and applications that we use in this paper to illustrate and evaluate our approach. In addition, we describe input data-sets and parameters that define system configurations. Finally, we motivate with one experiment the need for systematic optimization approaches.

\subsection{Heterogeneous computing systems}

Table 2 lists the major properties of heterogeneous computing systems Emil and Ida that we use for experimentation in this paper.

Ida comprises two Intel Xeon E5-2650 v4 general purpose CPUs on the host, and one GeForce GTX Titan X GPU as accelerator. Each CPU has 12 physical cores, and each core of CPU supports two threads. In total, the host CPUs provide 24 cores and 48 threads. The GPU device has 24 Streaming Multiprocessors (SM), and in total 3072 CUDA cores running at base frequency of $1 \mathrm{GHz}$.

Emil comprises two Intel Xeon E5-2695 v2 general purpose CPUs on the host, and one Intel Xeon Phi 7120P co-processing device. Similar to Ida, Each CPU of Emil has 12 cores that support two threads per core (known as logical cores) that amounts to a total of 48 threads. The Xeon Phi device has 61 cores running at $1.2 \mathrm{GHz}$ base frequency; each core supports four hardware threads. In total, the Xeon Phi supports 244 threads. The lightweight Linux operating system installed on the Xeon Phi device runs on one of the cores.

The parameters that define the system configuration for our optimization approach are shown in Table 3. All the parameters are discrete.

The considered system parameters for the Pearson Correlation Coefficient (PCC) application executed on Ida include the CPU workload and GPU workload. The workload fraction parameter can have any number in the range $\{0, . ., 100\}$, such that if $60 \%$ of the workload is assigned for processing to the host CPU, the remaining $100-60=40 \%$ is assigned to the GPU device.

The considered parameters for the pattern matching application executed on Emil include the number of CPU threads and accelerator threads, the CPU and accelerator thread affinity, and the CPU and accelerator workload fraction. The system parameter values for the host CPU threads are $\{12,24,36,48\}$, whereas for the accelerator are 


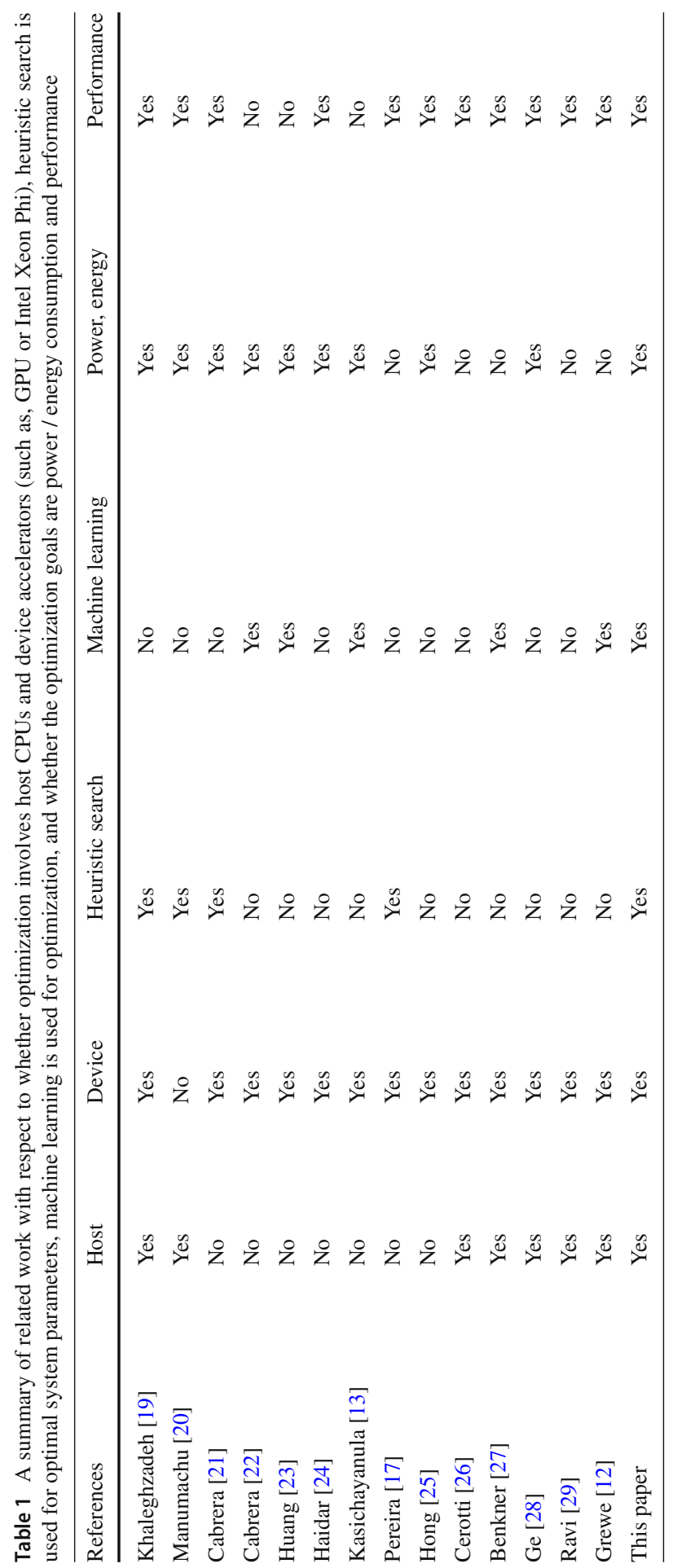


Table 2 Major features of Ida and Emil

\begin{tabular}{|c|c|c|c|c|}
\hline & \multicolumn{2}{|l|}{ Ida } & \multicolumn{2}{|l|}{ Emil } \\
\hline & Intel Xeon E5 & GeForce GPU & Intel Xeon E5 & Intel Xeon Phi \\
\hline Processor & E5-2650 v4 & GTX Titan X & E5-2695 v2 & $7120 \mathrm{P}$ \\
\hline Core frequency & $2.2-2.9 \mathrm{GHz}$ & $1-1.1 \mathrm{GHz}$ & $2.4-3.2 \mathrm{GHz}$ & $1.2-1.3 \mathrm{GHz}$ \\
\hline Number of cores & 12 & 3072 & 12 & 61 \\
\hline Number of threads & 24 & l & 24 & 244 \\
\hline Cache size & $30 \mathrm{MB}$ & I & $30 \mathrm{MB}$ & $30.5 \mathrm{MB}$ \\
\hline Mem. Bandwidth & $76.8 \mathrm{~GB} / \mathrm{s}$ & $336.5 \mathrm{~GB} / \mathrm{s}$ & $59.7 \mathrm{~GB} / \mathrm{s}$ & $352 \mathrm{~GB} / \mathrm{s}$ \\
\hline Memory size & 384 GB & $12 \mathrm{~GB}$ & $128 \mathrm{~GB}$ & $16 \mathrm{~GB}$ \\
\hline TDP & $105 \mathrm{~W}$ & $250 \mathrm{~W}$ & $115 \mathrm{~W}$ & $300 \mathrm{~W}$ \\
\hline
\end{tabular}

Table 3 The set of considered parameters and their values for our target systems

\begin{tabular}{lll}
\hline System & Parameters & Parameter values \\
\hline Ida & CPU workload fraction (CPU-W) & $\{0 \ldots 100\}$ \\
Emil & GPU workload fraction (GPU-W) & $\{100-$ CPU-W $\}$ \\
& CPU threads (CPU-T) & $\{12,24,36,48\}$ \\
& Accelerator threads (ACC-T) & $\{60,120,180,240\}$ \\
& CPU thread affinity (CPU-A) & $\{$ none (0), scatter (1), compact (2) $\}$ \\
& Accelerator thread affinity (ACC-A) & $\{$ balanced (0), scatter (1), compact (2) \\
& CPU workload fraction (CPU-W) & $\{0 \ldots 100\}$ \\
& Accelerator workload fraction (ACC-W) & $\{100-$ CPU-W $\}$ \\
\hline
\end{tabular}

$\{60,120,180,240\}$. The thread affinity can vary between none (0), compact (1), scatter (2)\} for the host CPUs, and \{balanced (0), compact (1), scatter (2)\} for the accelerator. Similar to the Emil system, the CPU and accelerator workload fraction for the Ida system can have values in the range $\{0, . ., 100\}$.

\subsection{Applications}

In this section, we describe two data-intensive applications that we have prepared for execution in hybrid mode (that is, the workload is shared between the host CPUs and the accelerating device): (1) Pearson Correlation Coefficient (PCC) and (2) parallel pattern matching. While the PCC application is adapted to GPU-accelerated systems, the pattern matching application targets systems accelerated with the Intel Xeon Phi.

\subsubsection{Pearson correlation coefficient (PCC)}

The Pearson correlation coefficient [30], which is also known as Pearson productmoment correlation, is a statistical method for measuring the strength of a relationship between two variables $x$ and $y$. The correlation may be positive (1), negative (-1), or 
there is no correlation (0). Positive correlation means that if variable $x$ is increased, there will be an increase on the $y$ variable, whereas the negative correlation means that an increase on $x$ would result with a decrease on $y$. A value of 0 for the correlation means that the two variables are not related and increase on one variable has no effect on the other one.

The strength of the relationship is measured using the absolute value of the correlation, for instance, $a b s(-0.23)=0.23$ is a stronger relationship than 0.2.

The Pearson correlation coefficient is calculated using the following formula [31]:

$$
\rho_{x y}=\frac{\left.\left.\sum_{i=1}^{n}\left(x_{i}-\bar{x}\right)\left(y_{i}-\bar{y}\right)\right)\right)}{\sqrt{\left.\sum_{i=1}^{n}\left(x_{i}-\bar{x}\right)^{2}\right)} \sqrt{\left.\sum_{i=1}^{n}\left(y_{i}-\bar{y}\right)^{2}\right)}}
$$

The Pearson coefficient correlation has a wide range of applications. For instance, [31] show its application to Magnetic Resonance Imaging (MRI) for analyzing the functional connectivity of different brain regions.

In this paper, we apply the Pearson coefficient correlation to rows of matrices. Note that for each row, we calculate the relationship with all other rows below in the matrix. We added the glue code to the CPU and GPU PCC implementations for enabling the hybrid execution. The input matrix is split row-wise between the CPUs on the host and GPU.

It is worth to mention, that for this specific application, a 50-50\% workload distribution does not mean that the work is equally divided between the host and the accelerator. This is because, for PCC the work depends not only on the amount of assigned rows of the matrix, but also the row-indices of the assigned rows. That is, rows at the top of matrix incur more work than those at the bottom. For instance row 0 compares itself with rows $1,2,3 \ldots n$. However, row 50 compares itself only with rows $51,52, \ldots n$.

\subsubsection{Parallel pattern matching}

Pattern matching is the process of finding multiple and overlapping occurrences of sub-strings in a string. Common uses of pattern matching are in search and replace functions, determining the location of a pattern, or highlighting important information out of huge data sets. Pattern matching is also used in intrusion detection systems (to identify potential threats) or computational biology (to identify the location of some patterns in large DNA sequences).

In this paper, we use an implementation of the pattern matching algorithm [32] based on the Aho-Corasick [33] algorithm and is tailored for application in bioinformatics. More specifically we use this algorithms to find sub-sequences of strings in the real-world human DNA sequence (3.17 GB), which are obtained from the GenBank sequence database of the National Center for Biological Information [34]. The algorithm is characterized by three main features including the thread-level parallelism, the SIMD-level parallelism, and the use of an optimized state transition table (STT), which helps reducing memory references at the cost of increasing the size of the STT. The basic idea is that the input string is first split among all available processing 
units (CPUs and accelerators), then each processing unit will further split the mapped input across the available cores/threads. The SIMD level parallelism is achieved in a similar manner, where the input mapped to a core is split into as many chunks as a processor can process SIMD instructions, then the SIMD transition function can operate on such chunks in parallel. Details regarding the design and implementation aspects of the pattern matching algorithm tailored for DNA sequence analysis can be found in [35].

Please note that the 50-50\% workload distribution of the pattern matching application means that the workload is equally divided between the host and the accelerator.

\subsection{Motivational experiment}

Heterogeneous computing systems may comprise several CPUs and accelerators. Optimal work-sharing among available CPUs and accelerators is not obvious. Furthermore, considering both execution time and energy consumption makes the work-sharing problem more complex.

Figure 2 illustrates this problem with the PCC application running on the Ida heterogeneous system that comprises two general-purpose CPUs and one GPU as accelerator. For simplicity, we only show the results for a particular input size (that is a matrix with 1024 rows and 8192 columns) and we only vary the parameter CPU Fraction (that is the number of rows of the matrix mapped to the CPU). Furthermore, we consider only CPU Fraction values that are product of 32 .

From this experiment, we may derive the following insights:

- When we consider the Throughput $[M B / s]$ only, the optimal value for the number of matrix rows assigned to the CPU is 160 and rest of the matrix rows is mapped to the GPU (Fig. 2a).

- When optimizing for the Power $[W]$ consumption, the execution on the CPU only is preferable (Fig. 2b); please note that the overhead of data transfers between CPU and GPU is considered for optimization.

- When optimizing for Energy Efficiency $[M B / J]$ that considers both the throughput and the power consumption, the optimal value for the number of matrix rows assigned to the CPU is 256 (Fig. 2c).

\section{Our approach for optimization of heterogeneous systems}

In this section, we describe our approach for optimization of heterogeneous computing systems that combines AI heuristic search techniques with machine learning.

\subsection{System optimization with Al heuristics and machine learning (AML)}

In this section, we propose an intelligent approach for the determination of nearoptimal system configuration using AI heuristic search techniques and machine learning. We contrast traditional approaches (also known as brute-force search) that we 


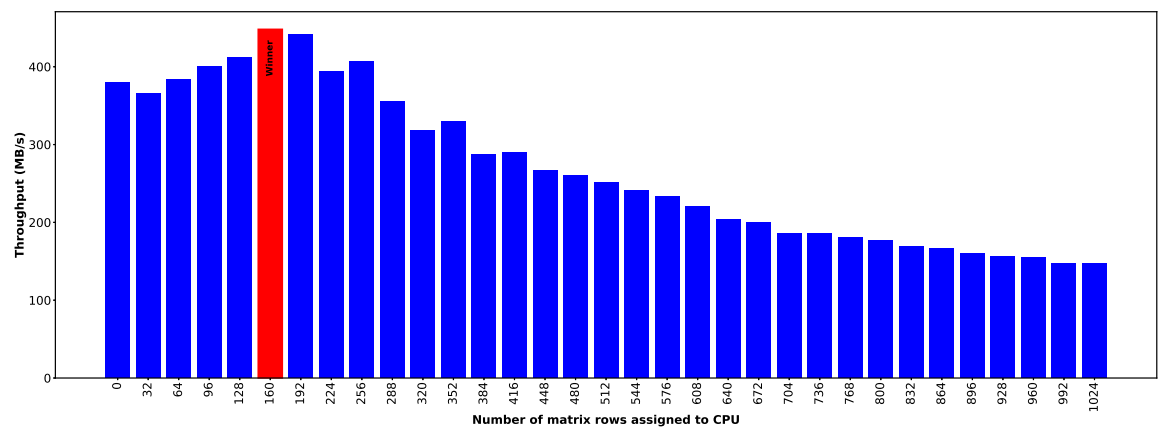

(a) Max throughput is achieved when 160 rows are mapped to CPU and the rest to GPU.

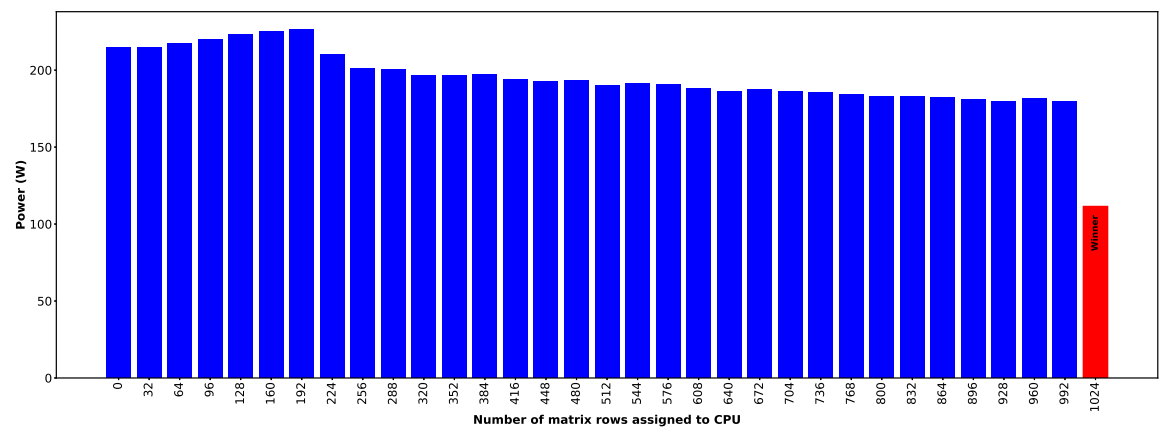

(b) All 1024 rows of matrix mapped to CPU results with the minimal power consumption. Data transfer between CPU and GPU is prohibitive.

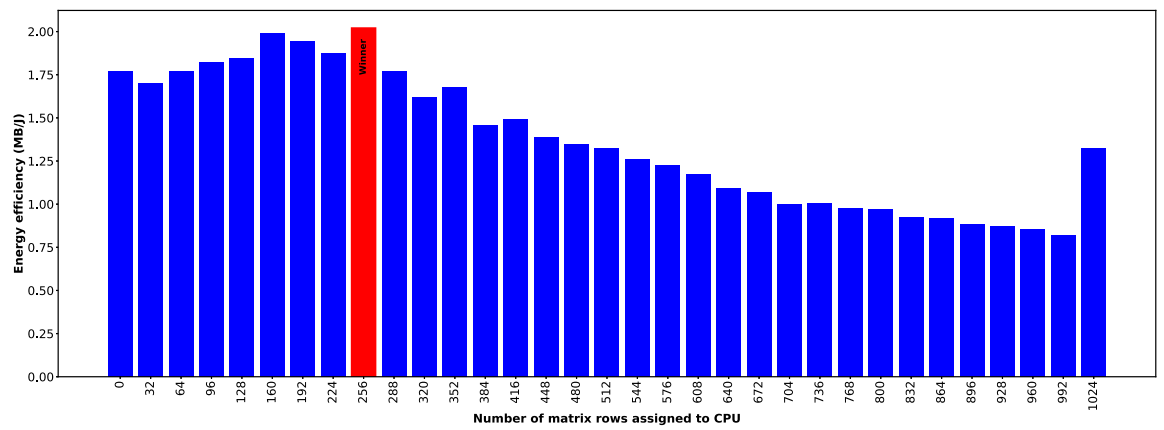

(c) Max energy efficiency is achieved when 256 rows are mapped to CPU and the rest to GPU.

Fig. 2 The workload fraction (i.e. number of matrix rows) assigned to the CPU that delivers the highest throughput $(\mathrm{MB} / \mathrm{s})$, lowest power consumption $(\mathrm{W})$, or highest energy efficiency $(\mathrm{MB} / \mathrm{J})$ is not the same. The figure shows the experiments for the PCC application running on Ida for matrix size $1024 \times 8192$. Measurements include also the performance overhead for data transfers between CPU and GPU

refer to as Enumeration and Measurements (EnuM), with our approach of combining AI heuristics with Machine Learning (AML).

The EnuM technique (also known, as brute-force search) is depicted in Fig. 3. Compartment Enumeration visualizes the process of selecting each feasible value of various system parameters. Thereafter, for each combination of parameter values 


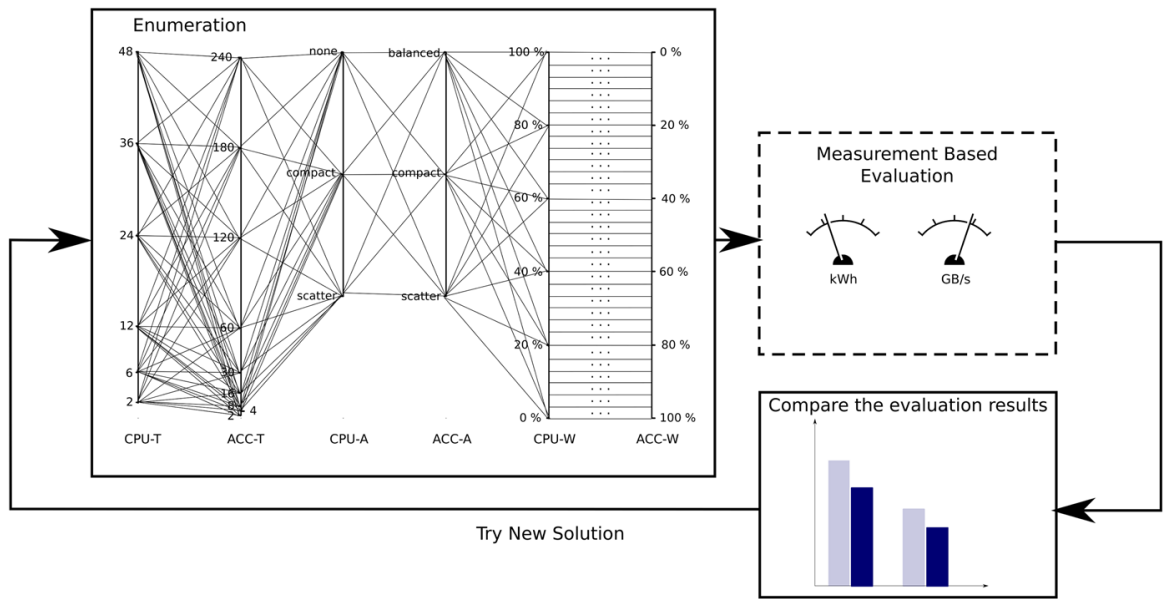

Fig. 3 Enumeration and measurements may help to determine the optimal system configuration, however the required effort may be high due to the large parameter space

the system performance is measured. Basically, EnuM involves program execution on a real computing system for all feasible system parameter values. For real-world programs and systems, EnuM may result with a very large number of performance measurement experiments. Furthermore, program execution on real computing systems may be time-consuming. Usually computing systems are shared among various users and it may be impractical to block other users for an unreasonable large amount of time while we execute our performance experiments. Key EnuM drawbacks include,

- a prohibitively large number of performance experiments,

- requires a dedicated access to the system under study.

Determining the optimal system configuration using brute-force may be prohibitively time expensive. The number of all possible system configurations is a product of parameter value ranges,

$$
\prod_{i=1}^{n} R_{c_{i}}=R_{c_{1}} \times R_{c_{2}} \times . . \times R_{c_{n}}
$$

where $C=\left\{c_{1}, c_{2}, \ldots, c_{n}\right\}$ is a set of parameters and each $c_{i}$ has a value range $R_{c_{i}}$.

The aim is to find an optimization approach that is able to find a near-optimal system configuration without having to measure the system performance for all feasible parameter values. Our approach AML is depicted in Fig. 4. Key AML features include,

- requires only a small fraction of possible performance experiments,

- uses a machine learning model for performance evaluation.

AML uses a AI heuristic search technique for parameter space exploration. The optimization process involves generation of system configuration based on random selection of parameter values and the system performance evaluation using a machine 


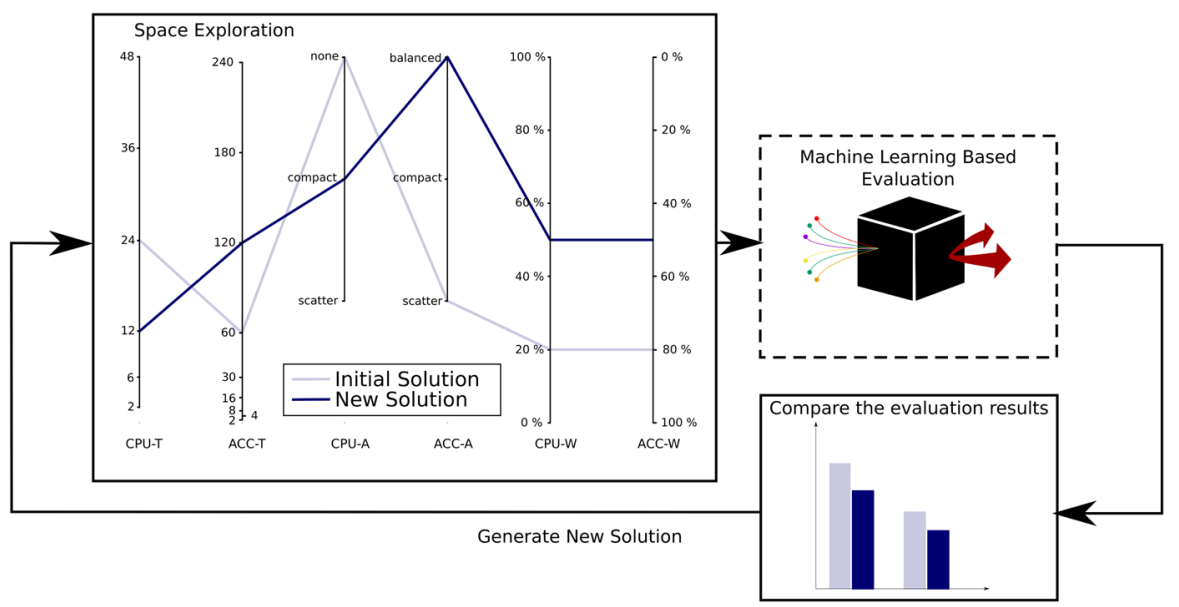

Fig. 4 Our approach uses an AI heuristic search technique for design space exploration and machine learning for system performance evaluation

learning model. AML guides the process of intelligent navigation through the parameter space towards the determination of the near-optimal system configuration. As a result, AML requires only a small fraction of possible performance experiments.

In what follows, we describe into more details the two most important components of our approach, which are the Simulated Annealing heuristic for design space exploration and boosted decision tree machine learning algorithm for system performance evaluation.

\subsubsection{Using simulated annealing (SA) for Parameter Space Exploration}

Simulated annealing (SA) [9] algorithm is a probabilistic optimization technique for approximately determining the global optimum of a function. SA is a popular technique for searching for optimum in a discrete space, where gradient-based methods are not applicable. An important feature of SA is the capability to find a global optimum also for functions that have many local optimums.

SA is inspired from the process of annealing in metallurgy, a technique that requires heating and controlled cooling of materials [8]. At high temperatures $T$ particles of the material have more freedom of movement, and as the temperature decreases the movement of particles is restricted. When the material is cooled slowly, the particles are ordered in the form of a crystal that represents minimal energy state of the material. 
Algorithm 1: Our SA-based algorithm for system configuration space exploration.

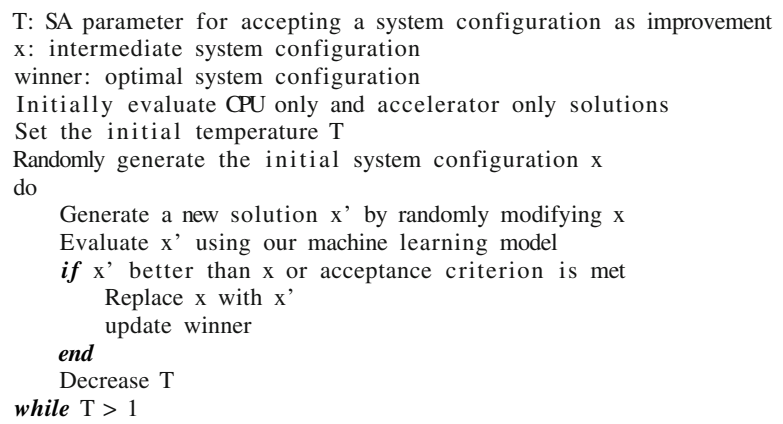

Algorithm 1 shows the pseudo-code of SA-based method for system configuration space exploration. A system configuration is determined by specific parameter values (see Table 3.) Since the optimal workload distribution for some of the experiments is to either run everything on the CPU or GPU, we initially evaluate the CPU and accelerator only system configurations (line 4). The process starts by initializing the temperature $T$ (line 5) and generating a random system configuration $x$ (line 6). New solutions $x^{\prime}$ are generated by randomly modifying the parameter values of current solution $x$ (line 8). We evaluate each of the generated system configurations using our machine learning based prediction model (line 9). If the performance per Joule of $x^{\prime}$ is better than the one of $x$, we replace $x$ with $x^{\prime}$ unconditionally (line 11), otherwise we consider accepting $x^{\prime}$ based on probability $p$ that is described by Equation 3 .

$$
p=\exp \left(\left(x-x^{\prime}\right) / T\right)
$$

The acceptance criterion (also known as Boltzmann's probability distribution [8]) allows simulated annealing to get out of local optimums in favor of a global optimum. The temperature variable plays a decisive role in the acceptance criterion. If the temperature $T$ is high, the system is more likely to accept solutions that are worse than the current one. After each new solution we decrease the temperature (line 14). Steps 8-14 are repeated as long as the temperature $T$ is greater than one (line 15).

Figure 5 illustrates the process of space exploration using SA for PCC application on Ida system (see Sect. 3.2.1). While there are many local optimums, our SA-based method manages to avoid them and finds the global optimum.

Our optimization goal is to maximize the performance per Joule, which is the rate of computation that can be delivered for every consumed watt. In HPC community, the standard metric for computationally-intensive application is the floating point operations per second (FLOPS). As we target data-intensive applications we use megabytes-per-second (MB/s) as a metric to indicate the application's throughput at run time. Beside the application performance we also consider the energy consumption, therefore the optimization goal metric is indicated by the total amount of data that can be processed for every consumed watt, that is megabytes-per-watt-second (MB/Ws). Section 5.1 introduces evaluation metrics. 


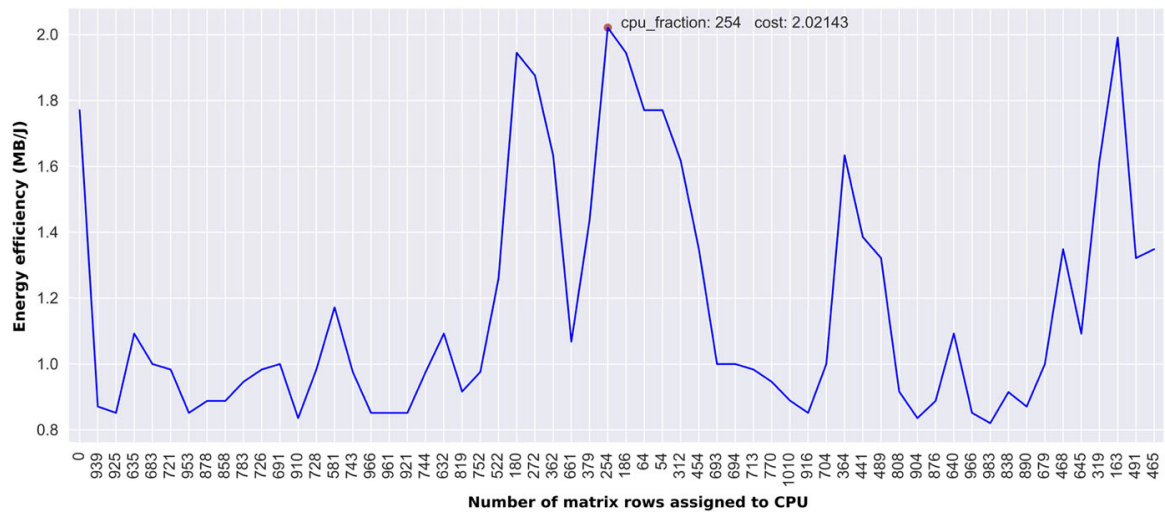

Fig. 5 An example of the configuration space exploration for PCC on Ida using our SA-based method. We may observe the presence of multiple local optimums, and that the SA determined the global optimum. The matrix size used for this experiment is $1024 \times 8192$

\subsubsection{Using machine learning for evaluation of system configuration}

In this section, we describe our supervised machine learning model that is used for evaluation of performance and energy consumption of data-parallel applications.

Our goal is to build a machine learning model that is able to predict the energy efficiency for a target data-parallel application running on target heterogeneous system based on characteristics of the given workload, and the available resources. Instead of using analytical models that are tightly coupled to a particular problem and environment, we use supervised machine learning that can be trained for various data-parallel programs and heterogeneous systems.

Preliminary results that compare various machine learning models (including linear regression, Poisson regression, and boosted decision tree regression) have suggested that for this particular type of problem the decision tree regression machine learning algorithm results with highest prediction scores. Hence, the decision tree regression is used in our approach.

We use the python science kit [36] (scikit-learn) to develop our machine learning model. The decision tree regression model is boosted with the Adaboost ensemble method, which results with further improvement with respect to the prediction accuracy.

We have executed the hybrid (CPU + accelerator) implementations of our selected application for all possible parameter values listed in Table 3. The energy efficiency for each execution is measured and recorded to the data-set, which constitutes the ground truth table. Note that for each possible values of the system configuration parameters the application has been executed multiple times $(40 \times 4)$ and the average values have been considered. The generated data set is used for training and validation. The set of parameters and the possible parameter values that our model is trained with is listed in Table 3.

To validate the results, we have used two types of well-established validation techniques, the $\mathrm{k}$-fold cross-validation [37] (where $k=10$ ) and the train test split technique 
(where $80 \%$ of the data is used for training and $20 \%$ of the data is used for testing). In both cases, the model resulted with high (more than 95\%) prediction accuracy scores, which we consider acceptable for the purpose of this study. Note that for evaluating the prediction model, we use the R squared [38] metric, also known as the coefficient of determination, which basically measures how close the data are to the fitted regression line. Also, note that there is still room for improvement with respect to the accuracy of the prediction model, for instance by tuning the hyper parameters, or providing more training data (especially for the data-set for the example of PCC running on GPU), which was out of scope of this paper.

The data pre-processing transforms the string-type input values (such as thread affinity, which value is balanced, scatter, or compact) into integer types (see Table 3 for the full set of parameters and their corresponding values).

\section{Evaluation}

In this section, we evaluate experimentally our proposed optimization approach for workload distribution on heterogeneous computing platforms.

\subsection{Evaluation metrics}

In this section, we describe a collection of metrics that we use for performance evaluation.

Time: is determined by the execution time of the slowest processing unit (that is, CPU or accelerator),

$$
\operatorname{Time}(s)=\max \left(C P U_{\text {Time }}, A C C_{\text {Time }}\right)
$$

$C P U_{\text {Time }}$ is workload processing time on CPU, and $A C C_{\text {Time }}$ is workload processing time on accelerator; $A C C_{\text {Time }}$ includes also the overhead for transferring data between CPU and accelerator.

Throughput: is the amount of data processed within a given time,

$$
\text { Throughput }(M B / s)=\text { Workload_size/Time }
$$

We can also express the throughput separately for each available processing unit as follows,

$$
\begin{aligned}
& C P U_{\text {Throughput }}(M B / s)=C P U_{W o r k l o a d \_s i z e} / C P U_{\text {Time }} \\
& A C C_{\text {Throughput }}(M B / s)=A C C_{\text {Workload_size }} / A C C_{\text {Time }}
\end{aligned}
$$

Energy: is the total energy (the static energy and the dynamic energy as defined in [19]) consumed by all used processing units. We use MeterPU [39] for energy measurement of host CPUs and GPUs, and x-MeterPU [40] for energy measurements on Intel Xeon Phi. Note that the performance and energy counters are initiated just 
before the function is called, i.e. we have wrappers around the function calls to start and stop the performance and energy counters. In the case when all the workload is mapped to the CPU the power consumption by the idle GPU is not considered in the measurements, whereas in cases where all the workload is mapped to the accelerator the the CPU power consumption is considered because the CPU is used for control and data management.

$$
\operatorname{Energy}(J)=C P U_{\text {Energy }}+A C C_{\text {Energy }}
$$

Power: is the consumed energy per time unit.

$$
\begin{gathered}
C P U_{\text {Power }}(W)=C P U_{\text {Energy }} / C P U_{\text {Time }} \\
A C C_{\text {Power }}(W)=A C C_{\text {Energy }} / A C C_{\text {Time }} \\
\operatorname{Power}(W)=C P U_{\text {Power }}+A C C_{\text {Power }}
\end{gathered}
$$

$C P U_{P o w e r}$ is power consumed by $\mathrm{CPU}, A C C_{\text {Power }}$ is power consumed by accelerator, and Power is the total power consumption for workload processing by CPU and accelerator.

Energy Efficiency: is the ratio of throughput to power,

$$
\text { Energy_Efficiency }(M B / J)=\operatorname{Throughput}(M B / s) / \operatorname{Power}(W)
$$

\subsection{Performance comparison of AML and EnuM}

In this section we,

- demonstrate experimentally the prediction accuracy of the ML-based performance model for Ida and Emil,

- demonstrate that it is significantly faster to evaluate the performance using the ML-based prediction model in comparison to the execution of the real program on Emil and Ida,

- demonstrate that AML is capable to determine a near-optimal system configuration with a small fraction of performance experiments in comparison to EnuM (that is, brute force search).

\subsubsection{Prediction accuracy and time-efficiency of the ML-based Performance Model}

To predict the energy efficiency of PCC application on Ida computing system, we have developed a prediction model based on Boosted Decision Tree Regression. In this section, we address two concerns: (1) are the model-based predicted results comparable to those obtained by measurements, and (2) how much faster is to evaluate the performance using the model compared to measurement?

Figure 6 depicts measured and predicted energy efficiency for PCC application on Ida system for various matrix sizes and number of matrix rows processed by the CPU. We may observe that there is good matching of results obtained from the model with the measurement results. 


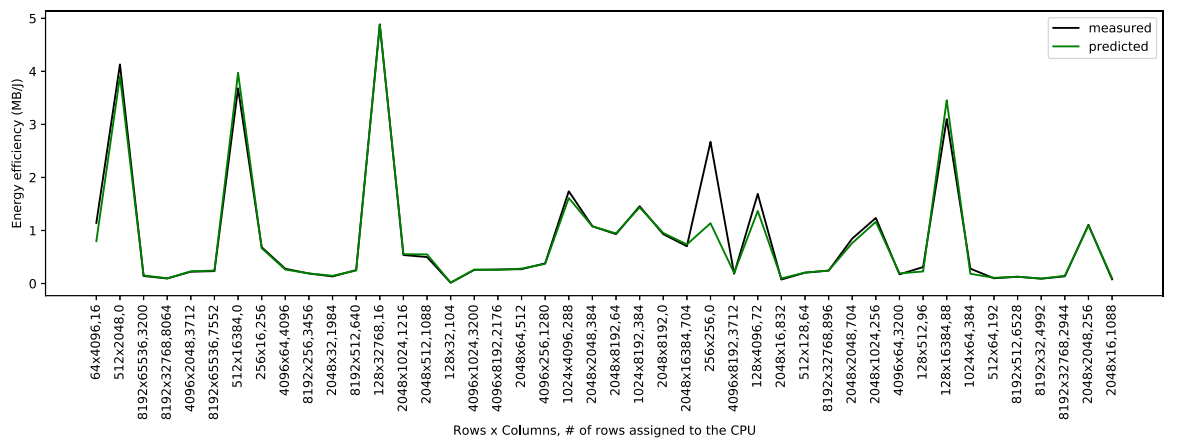

Fig. 6 Measured and predicted energy efficiency for PCC application on Ida system. Energy efficiency is predicted for various matrix sizes and number of matrix rows processed by CPU using our prediction model built based on Boosted Decision Tree Regression

EnuM involves running PCC on Ida to determine energy efficiency for various parameter values. Execution of 2912 EnuM experiments in this study took 13,404 seconds (approximately $3.7 \mathrm{~h}$ ). In contrast to EnuM, AML uses a machine learning model to predict energy efficiency for various parameter values; model-based evaluation lasts 3.5 milliseconds. Regarding time-efficiency, for 2912 performance experiments EnuM and AML compare as follows,

- EnuM: 13404 [s]

- AML: 10 [s]

We may observe that in this study AML is more than 1300 times faster than EnuM.

\subsubsection{Determining near-optimal system configuration for PCC on Ida}

In this section, we compare AML with EnuM with respect to search for a near-optimal system configuration for PCC application on Ida computing system. The optimization goal is the energy efficiency. Major properties of PCC and Ida are described in Sect. 3. AML and EnuM optimization techniques are described in Sect. 4.1.

We performed experiments for various numbers of matrix rows $(16,32,64,128$, 256, 512, 1024, 2048, 4096, 8192) and columns (16, 32, 64, 128, 256, 512, 1024, $2048,4096,8192,16384,32768,65536)$. The matrix elements are initialized with random values of float type. Note that the performance results reported in this section do not include the effort required to initialize the matrix. Considering parameter values ranges in this study there are,

- 14677 AML experiments, and

- 212914 EnuM experiments.

Figure 7 depicts examples of estimation of near-optimal CPU fraction with AML and EnuM for PCC application on Ida for various matrix sizes. CPU Fraction is the percentage of matrix rows that is mapped for processing to CPU; the rest of matrix is processed by GPU. The optimization goal is the energy efficiency (see Sect. 5.1).

Please note that Fig. 7 shows only examples of results where the CPU fraction determined by AML differs from the one determined by EnuM; that is only 24 matrix 


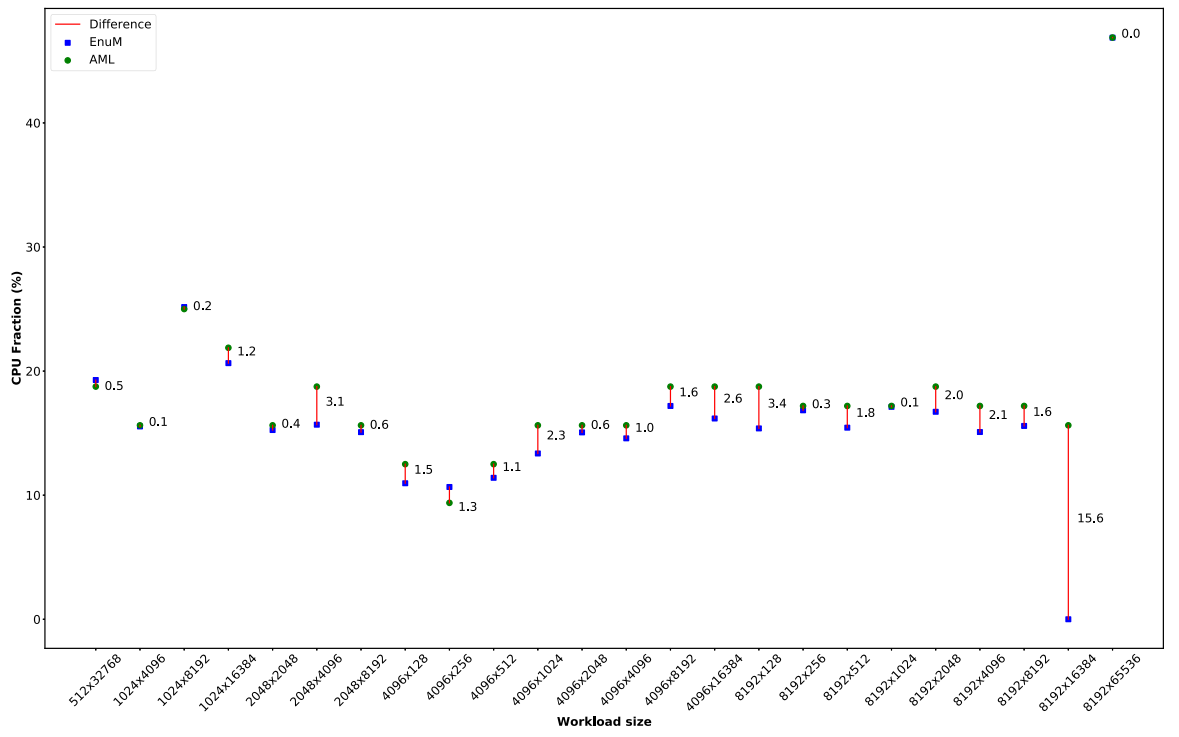

Fig. 7 Examples of estimation of near-optimal CPU Fraction with AML and EnuM for PCC application on Ida. CPU Fraction is the percentage of matrix rows that is mapped for processing to CPU. The optimization goal is the energy efficiency

sizes out of 130 in total. The data points depicted as small rectangles correspond to the EnuM results, whereas the data points depicted as small circles show the AML values. The vertical red lines show the difference between AML and EnuM.

We may observe that in most of the cases AML can determine near-optimal CPU fraction that yields the energy efficiency that is comparable to the one determined by EnuM. For 106 matrix sizes there was no difference between AML and EnuM.

The highest observed difference is $15.6 \%$ in the case of matrix size $8192 \times 16,384$; the EnuM suggests to map all the matrix rows to the GPU (that is, CPU fraction is 0 ), but the AML suggests to map $15.6 \%$ of the workload to the CPU and the rest to the GPU . While the CPU fraction difference of $15.6 \%$ is substantial, the difference between AML and EnuM with respect to the achieved optimization goal (that is, energy efficiency) is insignificant:

- AML achieved energy efficiency: $0.252(\mathrm{MB} / \mathrm{J})$

- EnuM achieved energy efficiency: $0.255(\mathrm{MB} / \mathrm{J})$

The corresponding energy efficiency results for workload sizes depicted in the Fig. 7 are listed in Table 4. We may observe that the difference between EnuM and AML with respect to energy efficiency is not significant; that is AML is capable of determining system configurations that result with energy efficiency comparable to EnuM. 
Table 4 Examples of achieved energy efficiency with EnuM and AML for various workload sizes of PCC application on Ida

\begin{tabular}{llll}
\hline Workload: Matrix $(\mathrm{R} \times \mathrm{C})$ & EnuM & AML & $\mid$ Difference $\mid$ \\
\hline $512 \times 32,768$ & 3.169 & 3.169 & 0.00000 \\
$1024 \times 4096$ & 2.072 & 2.067 & 0.00474 \\
$1024 \times 81,92$ & 2.021 & 2.021 & 0.00000 \\
$1024 \times 16,384$ & 1.750 & 1.744 & 0.00552 \\
$2048 \times 2048$ & 1.186 & 1.076 & 0.10936 \\
$2048 \times 4096$ & 1.079 & 1.059 & 0.01982 \\
$2048 \times 8192$ & 0.994 & 0.990 & 0.00362 \\
$4096 \times 128$ & 0.439 & 0.433 & 0.00587 \\
$4096 \times 256$ & 0.633 & 0.561 & 0.07285 \\
$4096 \times 512$ & 0.602 & 0.589 & 0.01367 \\
$4096 \times 1024$ & 0.597 & 0.580 & 0.01656 \\
$4096 \times 2048$ & 0.569 & 0.547 & 0.02169 \\
$4096 \times 4096$ & 0.529 & 0.523 & 0.00684 \\
$4096 \times 8192$ & 0.522 & 0.505 & 0.01669 \\
$4096 \times 16384$ & 0.487 & 0.487 & 0.00034 \\
$8192 \times 128$ & 0.233 & 0.211 & 0.02153 \\
$8192 \times 256$ & 0.252 & 0.243 & 0.00980 \\
$8192 \times 512$ & 0.280 & 0.268 & 0.01191 \\
$8192 \times 1024$ & 0.298 & 0.276 & 0.02204 \\
$8192 \times 2048$ & 0.279 & 0.270 & 0.00907 \\
$8192 \times 4096$ & 0.268 & 0.260 & 0.00828 \\
$8192 \times 8192$ & 0.267 & 0.256 & 0.01145 \\
$8192 \times 16,384$ & 0.255 & 0.252 & 0.00338 \\
$8192 \times 65,536$ & 0.529 & 0.529 & 0.00000 \\
\hline
\end{tabular}

\subsubsection{Determining near-optimal system configuration for parallel pattern matching on Emil}

In this section, we compare AML with EnuM with respect to search for a near-optimal system configuration for parallel pattern matching on Emil computing system. The optimization goal is the energy efficiency. We have described parallel pattern matching and Emil computing system in Sect. 3.

In this study, despite the fact that we tested only what we considered reasonable parameter values (listed on Table 3 in Sect. 3.1), 5590 experiments were required when we used EnuM. Our heuristic-guided approach AML that is based on Simulated Annealing and Machine Learning leads to comparatively good performance results, while requiring only a relatively small set of experiments to be performed.

Figure 8 depicts the energy efficiency of the pattern matching application when running using the system configuration suggested by AML. The solid horizontal line indicates the energy efficiency of the system configuration determined by EnuM. EnuM 


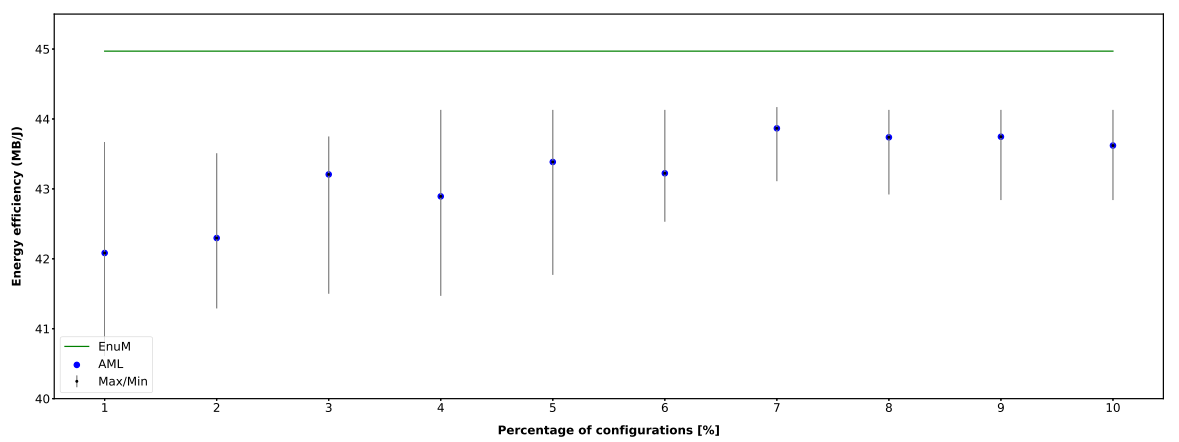

Fig. 8 Comparison of AML with EnuM for various percentages of possible system configurations of Emil. Note that the total number of configurations is 5590 and by trying only about $7 \%$ of all configurations using AML we manage to find a near optimal system configuration that delivers up to $97.55 \%$ of the energy efficiency that is determined by the EnuM by trying all possible configurations

finds the optimal solution by a kind of brute-force search. We may observe that after evaluating only about $7 \%$ of the possible configurations, AML is able to determine a system configuration that results with energy efficiency that is close to the one of the system configuration determined by EnuM. The difference between AML and EnuM with respect to the achieved optimization goal (that is, energy efficiency) is as follows:

- AML achieved energy efficiency: $43.87(\mathrm{MB} / \mathrm{J})$

- EnuM achieved energy efficiency: $44.97(\mathrm{MB} / \mathrm{J})$

Please note that AML uses a stochastic search algorithm for global optimization and to avoid ending at a local optima during the search sometimes it accepts a worse system configuration (see Sect. 4.1.1).

\section{Summary and future work}

Heterogeneous architectures are a viable way of building computer systems with a high peak performance and a lower energy consumption. Optimal work-sharing among available CPUs and accelerators is not obvious, and considering both performance and energy consumption further complicates the work-sharing problem. We have presented an approach that uses a probabilistic heuristic search technique for parameter space exploration. The optimization process involves generation of system configuration based on random selection of parameter values and the system performance evaluation using a machine learning model. We have evaluated our approach experimentally on heterogeneous systems that are accelerated with the Intel Xeon Phi or GPU for two applications: Pearson correlation coefficient and parallel pattern matching. In our experiments we have observed that,

- the more than $95 \%$ accuracy of the developed machine learning model enables to inform the search for optimal system configuration,

- by considering only about $7 \%$ of the possible system configurations our method finds a near-optimal configuration with respect to performance per Joule; 
- our method is 1300x faster than the brute-force search combined with measurement of program execution;

- applicability of our approach is not limited to one type of accelerator or application; the model can be trained for various accelerated systems.

In the context of edge, fog, and cloud computing, the data movement between the various layers is expensive, and it is not trivial to determine at which layer would be optimal to perform the processing. Furthermore, similarly to heterogeneous systems, it is expected that for particular problems, sharing the workload across the various resources available in the different layers might yield to an optimal solution with respect to energy, performance, and/or cost. Future work will study work-sharing in the context of the Edge, Fog and Cloud systems.

Funding Open access funding provided by Blekinge Institute of Technology.

Open Access This article is licensed under a Creative Commons Attribution 4.0 International License, which permits use, sharing, adaptation, distribution and reproduction in any medium or format, as long as you give appropriate credit to the original author(s) and the source, provide a link to the Creative Commons licence, and indicate if changes were made. The images or other third party material in this article are included in the article's Creative Commons licence, unless indicated otherwise in a credit line to the material. If material is not included in the article's Creative Commons licence and your intended use is not permitted by statutory regulation or exceeds the permitted use, you will need to obtain permission directly from the copyright holder. To view a copy of this licence, visit http://creativecommons.org/licenses/by/4.0/.

\section{References}

1. Dongarra JJ, Meuer HW, Strohmaier E et al (2020) TOP500 Supercomputer Sites. http://www.top500. org/. Accessed Jan. 2021

2. Czarnul P (2018) Parallel programming for modern high performance computing systems. CRC Press, Boca Raton

3. Markidis S, Chien S, Laure E, Peng I, Vetter J (2018) NVIDIA tensor core programmability, performance precision. In: 2018 IEEE international parallel and distributed processing symposium workshops (IPDPSW), pp 522-531. https://doi.org/10.1109/IPDPSW.2018.00091

4. Amaral V, Norberto B, Goulão M, Aldinucci M, Benkner S, Bracciali A, Carreira P, Celms E, Correia L, Grelck C, Karatza H, Kessler C, Kilpatrick P, Martiniano H, Mavridis I, Pllana S, Respício A, Simão J, Veiga L, Visa A (2020) Programming languages for data-intensive HPC applications: a systematic mapping study. Parallel Comput 91(102):584. https://doi.org/10.1016/j.parco.2019.102584

5. Mittal S, Vetter JS (2015) A Survey of CPU-GPU Heterogeneous Computing Techniques. ACM Comput Surv 47(4):69:1-69:35, https://doi.org/10.1145/2788396

6. Pllana S, Xhafa F (2017) Programming multicore and many-core computing systems. Wiley, Hoboken. https://doi.org/10.1002/9781119332015

7. Alba E (2018) How can metaheuristics help software engineers? In: Colanzi TE, McMinn P (eds) Search-based software engineering. Springer, Cham, pp 89-105

8. Press WH, Teukolsky SA, Vetterling WT, Flannery BP (2007) Numerical recipes 3rd edition: the art of scientific computing, 3rd edn. Cambridge University Press, New York

9. Kirkpatrick S, Gelatt D, Vecchi M (1983) Optimization by Simulated Annealing. Science 220(4598):671-680. https://doi.org/10.1126/science.220.4598.671

10. Karaboga D, Basturk B (2007) Artificial bee colony (ABC) optimization algorithm for solving constrained optimization problems. In: Proceedings of the 12th international fuzzy systems association world congress on foundations of fuzzy logic and soft computing. Springer, Berlin. IFSA 07, pp 789-798 
11. Memeti S, Pllana S, Binotto A, Kołodziej J, Brandic I (2018) Using meta-heuristics and machine learning for software optimization of parallel computing systems: a systematic literature review. Computing. https://doi.org/10.1007/s00607-018-0614-9

12. Grewe D, O'Boyle MF (2011) A static task partitioning approach for heterogeneous systems using OpenCL. In:Jens k (ed) Compiler construction, Springer,Berlin, Heidelberg pp 286-305

13. Kasichayanula K, Terpstra D, Luszczek P, Tomov S, Moore S, Peterson GD (2012) (2012) Power aware computing on GPUS. In: Application accelerators in high performance computing (SAAHPC). Symposium on, IEEE, pp 64-73

14. Fahringer T, Jugravu A, Pllana S, Prodan R, Seragiotto C Jr, Truong HL (2005) Askalon: a tool set for cluster and grid computing. Concurr Comput Pract Exp 17(2-4):143-169. https://doi.org/10.1002/ cpe.929

15. Grzonka D, Jakóbik A, Kołodziej J, Pllana S (2018) Using a multi-agent system and artificial intelligence for monitoring and improving the cloud performance and security. Futur Gener Comput Syst 86:1106-1117. https://doi.org/10.1016/j.future.2017.05.046

16. Xhafa F, Gonzalez JA, Dahal KP, Abraham A (2009) A ga(ts) hybrid algorithm for scheduling in computational grids. In: Corchado E, Wu X, Oja E, Herrero Á, Baruque B (eds) Hybrid Artificial Intelligence Systems. Springer, Berling, pp 285-292

17. Pereira C, Pinheiro A, Schirru R (2020) Automatic block dimensioning on GPU-accelerated programs through particle swarm optimization. Inf Softw Technol 123(106):299. https://doi.org/10.1016/j.infsof. 2020.106299

18. Memeti S, Pllana S (2016) Combinatorial optimization of work distribution on heterogeneous systems. In: 45th international conference on parallel processing workshops (ICPPW), pp 151-160. https://doi. org/10.1109/ICPPW.2016.35

19. Khaleghzadeh H, Fahad M, Shahid A, Manumachu RR, Lastovetsky A (2021) Bi-objective optimization of data-parallel applications on heterogeneous HPC platforms for performance and energy through workload distribution. IEEE Trans Parallel Distrib Syst 32(3):543-560. https://doi.org/10.1109/TPDS. 2020.3027338

20. Manumachu RR, Lastovetsky A (2018) Bi-objective optimization of data-parallel applications on homogeneous multicore clusters for performance and energy. IEEE Trans Comput 67(2):160-177. https://doi.org/10.1109/TC.2017.2742513

21. Cabrera A, Acosta A, Almeida F, Blanco V (2019) A heuristic technique to improve energy efficiency with dynamic load balancing. J Supercomput 75(3):1610-1624. https://doi.org/10.1007/s11227-0182718-6

22. Cabrera A, Almeida F, Blanco V, Castellanos-Nieves D (2019) Improving energy consumption in iterative problems using machine learning. In: International conference on parallel processing and applied mathematics. Springer, Cham, pp 134-143

23. Huang Y, Guo B, Shen Y (2019) Gpu energy consumption optimization with a global-based neural network method. IEEE Access 7:64303-64314. https://doi.org/10.1109/ACCESS.2019.2915380

24. Haidar A, Jagode H, YarKhan A, Vaccaro P, Tomov S, Dongarra J (2017) Power-aware computing: Measurement, control, and performance analysis for Intel Xeon Phi. In: 2017 IEEE high performance extreme computing conference (HPEC), pp 1-7. https://doi.org/10.1109/HPEC.2017.8091085

25. Hong S, Kim H (2010) An integrated gpu power and performance model. In: ACM SIGARCH computer architecture news, vol 38. ACM, pp 280-289

26. Cerotti D, Gribaudo M, Iacono M, Piazzolla P (2016) Modeling and analysis of performances for concurrent multithread applications on multicore and graphics processing unit systems. Concurr Comput Pract Exp 28(2):438-452. https://doi.org/10.1002/cpe.3504

27. Benkner S, Pllana S, Traff J, Tsigas P, Dolinsky U, Augonnet C, Bachmayer B, Kessler C, Moloney D, Osipov V (2011) PEPPHER: efficient and productive usage of hybrid computing systems. Micro IEEE 31(5):28-41

28. Ge R, Feng X, Burtscher M, Zong Z (2014) Performance and energy modeling for cooperative hybrid computing. In: 20149th IEEE international conference on networking, architecture, and storage (NAS), pp 232-241. https://doi.org/10.1109/NAS.2014.42

29. Ravi VT, Agrawal G (2011) A dynamic scheduling framework for emerging heterogeneous systems. In: 2011 18th international conference on high performance computing (HiPC). IEEE, pp 1-10

30. Boslaugh S (2012) Statistics in a nutshell: a desktop quick reference. O'Reilly Media Inc.” 
31. Eslami T, Awan MG, Saeed F (2017) Gpu-pcc: A gpu based technique to compute pairwise Pearson's correlation coefficients for big FMRI data. In: Proceedings of the 8th ACM international conference on bioinformatics, computational biology, and health informatics, pp 723-728

32. Memeti S, Pllana S (2015) Accelerating dna sequence analysis using intel(r) xeon phi(tm). In: 2015 IEEE Trustcom/BigDataSE/ISPA, vol 3. IEEE, pp 222-227. https://doi.org/10.1109/Trustcom.2015. 636

33. Aho AV, Corasick MJ (1975) Efficient string matching: an aid to bibliographic search. Commun ACM 18(6):333-340

34. NCBI (2015) National Center for Biotechnology Information U.S. National Library of Medicine. http:// www.ncbi.nlm.nih.gov/genbank. Accessed: Dec. 2015

35. Memeti S, Pllana S (2018) A machine learning approach for accelerating DNA sequence analysis. Int J High Perform Comput Appl 32(3):363-379

36. Pedregosa F, Varoquaux G, Gramfort A, Michel V, Thirion B, Grisel O, Blondel M, Prettenhofer P, Weiss R, Dubourg V et al (2011) Scikit-learn: machine learning in python. J Mach Learn Res 12:28252830

37. Anguita D, Ghelardoni L, Ghio A, Oneto L, Ridella S (2012) The'k' in k-fold cross validation. In: ESANN

38. Renaud O, Victoria-Feser MP (2010) A robust coefficient of determination for regression. J Stat Plan Inference 140(7):1852-1862

39. Lu Li, Christoph Kessler (2016) MeterPU: measurement abstraction API enabling energy-tuned skeleton backend selection. J Supercomput. https://doi.org/10.1007/s11227-016-1792-X

40. Memeti S, Li L, Pllana S, Kolodziej J, Kessler C (2017) Benchmarking OpenCL, OpenACC, OpenMP, and CUDA: programming productivity, performance, and energy consumption. In: Proceedings of the 2017 workshop on adaptive resource management and scheduling for cloud computing. ACM, New York ARMS-CC '17, pp 1-6. https://doi.org/10.1145/3110355.3110356

Publisher's Note Springer Nature remains neutral with regard to jurisdictional claims in published maps and institutional affiliations. 